\title{
Anabases
}

ANABASES Traditions et réceptions de l'Antiquité

$24 \mid 2016$

Varia

\section{Les couples étrusques au banquet : pratiques rituelles et alimentaires}

\section{Laurent Hugot}

\section{OpenEdition}

Journals

Édition électronique

URL : http://journals.openedition.org/anabases/5887

DOI : 10.4000/anabases.5887

ISSN : 2256-9421

Éditeur

E.R.A.S.M.E.

Édition imprimée

Date de publication : 10 novembre 2016

Pagination : 77-91

ISSN : 1774-4296

\section{Référence électronique}

Laurent Hugot, "Les couples étrusques au banquet: pratiques rituelles et alimentaires », Anabases [En ligne], 24 | 2016, mis en ligne le 01 octobre 2019, consulté le 21 décembre 2020. URL : http:// journals.openedition.org/anabases/5887 ; DOI : https://doi.org/10.4000/anabases.5887

(c) Anabases 
Anabases 24 (2016), p. 77-91.

\title{
Les couples étrusques au banquet : pratiques rituelles et alimentaires
}

Laurent Hugot

\begin{abstract}
L
es deux superbes sarcophages de terre cuite dits des Époux, conservés pour l'un au Musée du Louvre et pour le second au Musée de la Villa Giulia à Rome, sont devenus les images emblématiques des couples étrusques et même audelà, de la civilisation et du mode de vie des Étrusques. Il existe cependant de nombreuses autres belles figurations de couples, tout au long de l'histoire des anciens toscans. Parmi elles, les deux représentations des couples de la tombe des Boucliers, sont remarquables'. Le premier groupe (fig. 1), constitué de Velia Seitithi et de Larth Velcha correspond, selon Massimo Pallottino, aux fondateurs de la tombe familiale, ayant vécu dans la première moitié du III ${ }^{\mathrm{e}}$ s. av. J.-C.. ${ }^{2}$. Le second couple (fig. 2) est constitué de Ravnthu Aprthnai et Velthur Velcha, qui auraient vécu à la fin du $\mathrm{IV}^{\mathrm{e}}$ ou au début du $\mathrm{III}^{\mathrm{e}} \mathrm{s}$. av. J.-C. et qui seraient les parents de Larth ${ }^{3}$. Les fresques de cette tombe sont extraordinaires par de nombreux aspects. Par la qualité des peintures, par les inscriptions qui nous permettent de mieux connaître cette famille aristocratique tarquinienne et en particulier Larth ;
\end{abstract}

1 S. Steingräвer, Catalogo ragionato della pittura etrusca, Milan, 1984, p. 346-347, pl. 145-149 (avec la bibliographie antérieure); A. Morandi, "La tomba degli Scudi di Tarquinia. Contributo epigrafico per l'esegesi dei soggetti ", Mélanges de l'École Française de Rome. Antiquité 99-1 (1987), p. 95-110; http://www.persee.fr/doc/ mefr_0223-5102_1987_num_99_1_1538 (site consulté le 17 février 2016). Sur la famille, voir aussi M. Morandi Tarabella, Prosopografia Etrusca. I. Corpus. 1 Etruria Meridionale, Rome, 2004, p. 179-186.

2 Stefan Steingräber a depuis remonté un peu la datation de la tombe à la fin du $I^{\mathrm{e}} \mathrm{s}$. av. J.-C. Steingräber, Catalogo ragionato, p. 346-347.

3 M. Pallottino, “ Tarquinia », Monumenti antichi 36 (1937), col. 1-162 et pl. 1-10. 


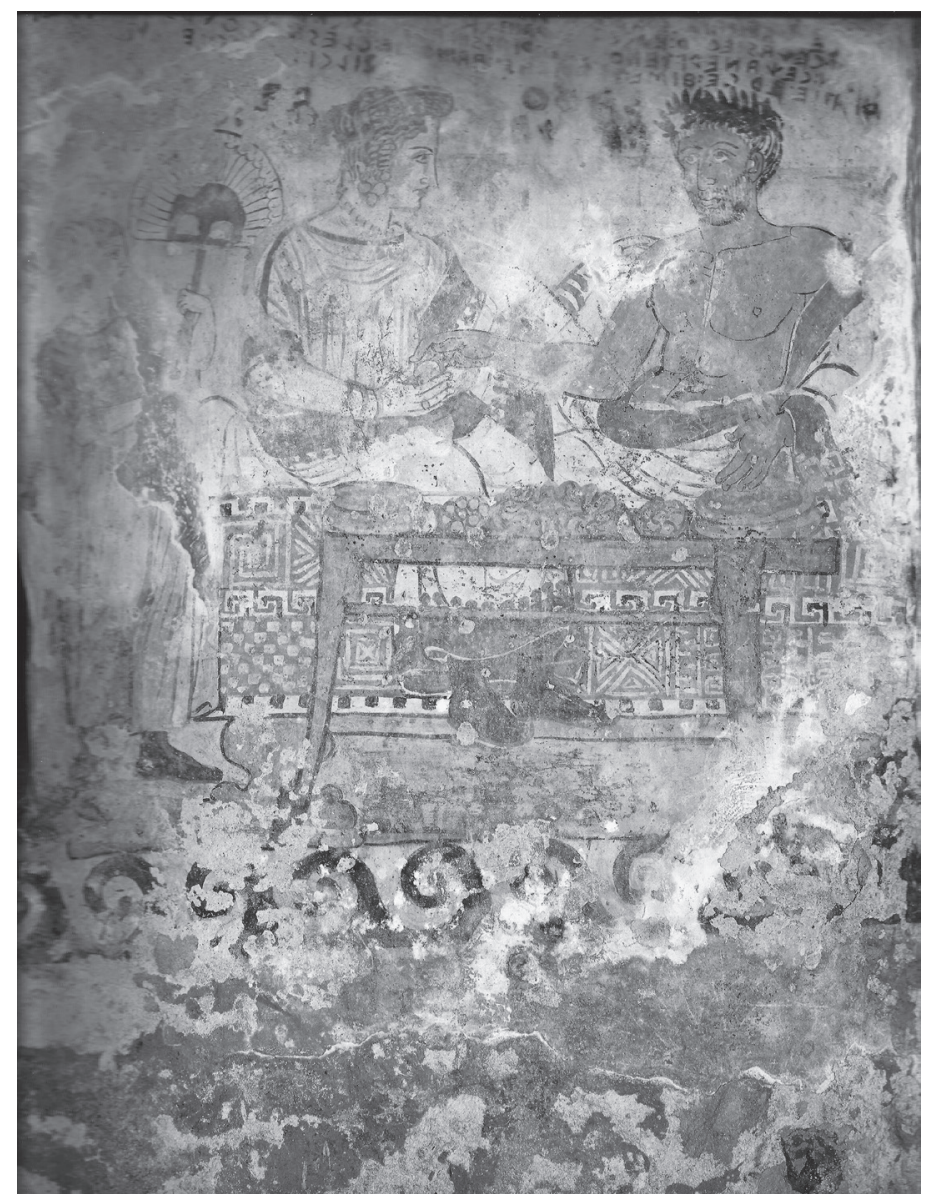

Fig. 1. Tarquinia, Tombe des Boucliers, Velia Seitithi et Larth Velcha.

D’après S. Steingräвer, Catalogo ragionato della pittura etrusca, Milan, 1984, p. 146.

mais aussi par les nombreux détails représentés par les artistes sur l'ensemble des fresques, en particulier le soin porté à peindre la nourriture posée sur les tables devant les klinai. La représentation de personnages devant des tables garnies n'est pas fréquente en Étrurie et quand c'est le cas, il est rare qu'on puisse clairement distinguer de quels aliments il s'agit. Pour Catulle, qui utilise à l'envi des archétypes grossiers, l'Ombrien était économe et l'Étrusque, lui, était obèse ! Pourtant, nous avons bien peu de représentations de nourriture pour un peuple qui était censé passer une grande partie de ses journées à manger sans retenue ${ }^{4}$.

4 Catulle XXXIX, Contre Égnatius. 


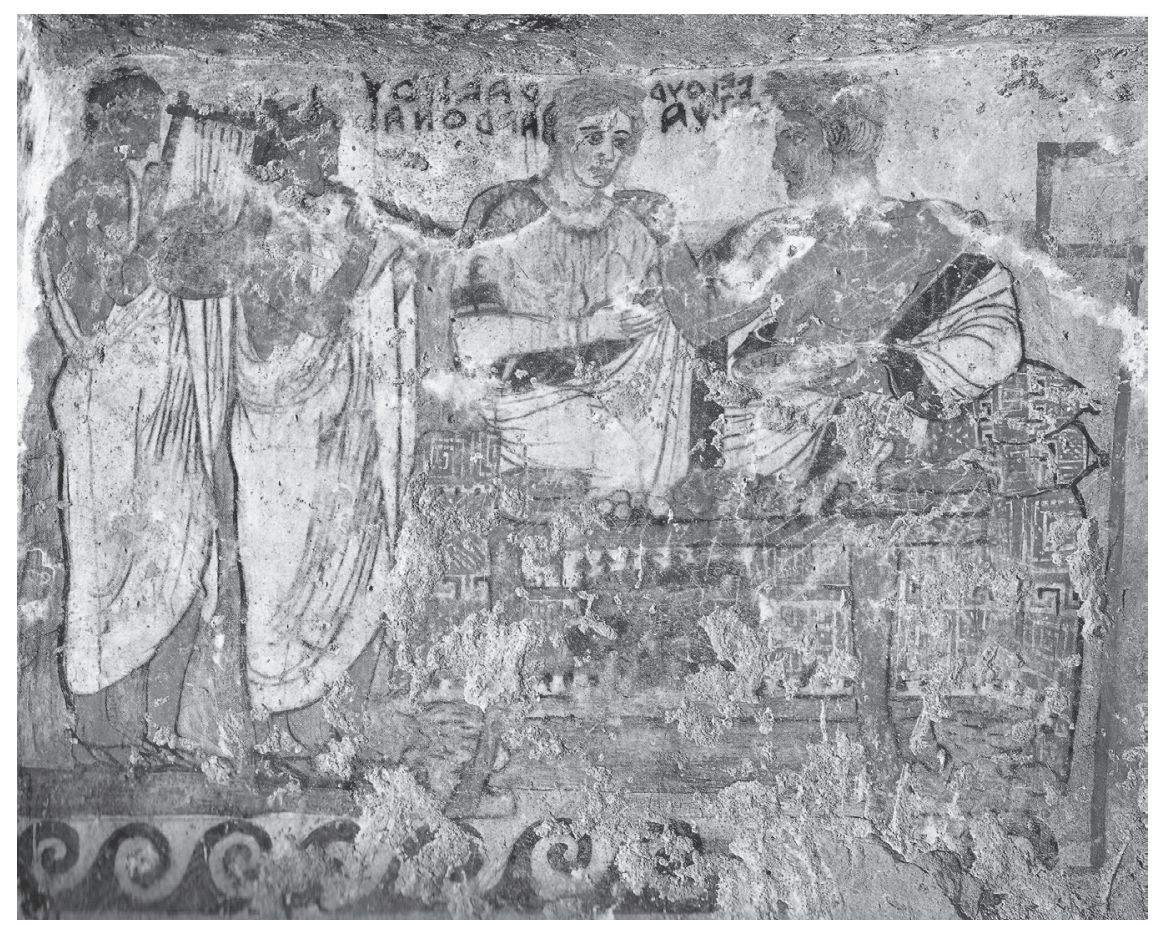

Fig. 2. Tarquinia, Tombe des Boucliers, Ravnthu Aprthnai et Velthur Velcha.

D’après M. Monetri, Pittura etrusca in Tarquinia, Milan, 1974, p. 121.

La plus ancienne représentation d'un banquet avec des victuailles déposées sur une table se trouve sur le couvercle de l'ossuaire de terre cuite du milieu du VII ${ }^{\mathrm{e}}$ s. av. J.-C., retrouvé dans une tombe à puits à Montescudaio, qui est conservé au Musée archéologique de Florence (fig. 3). Il s'agit d'un banquet assis, dans la tradition orientale, probablement assyrienne, dans un univers stylistique et culturel villanovien ${ }^{5}$. On est aussi proche de la description homérique du banquet d'Alcinoos ${ }^{6}$. Ici, pas de couple au banquet : l'homme est assis seul sur un trône,

5 Voir Principi etruschi tra Mediterraneo ed Europa, (catalogue de l'exposition de Bologne, Museo Civico Archeologico 2001), Venise, 2001, p.199-200, n 206. Sur le banquet assis en Étrurie : A. S. Tuck, « The Etruscan Seated Banquet: Villanovan Ritual and Etruscan Iconography », American Journal of Archaeology 98 (1994), p. 617-628. Sur l'ossuaire, F. Magi, "L'ossuario di Montescudaio », in Atti del Primo simposio internazionale di protostoria italiana, Rome, 1969, p. 121-133; F. Nicosia, “ Il cinerario di Montescudaio : proposta per un nuovo restauro », Studi Etruschi 37 (1969), p. 369-401; G. BARToloni, “ Il cinerario di Montescudaio e il culto degli antenati », in Francesco Nicosia. L'archeologo e il soprintendente. Scritti in memoria, Florence, 2012, p. 91-96.

6 Homère, Odyssée VII, 167-232. 


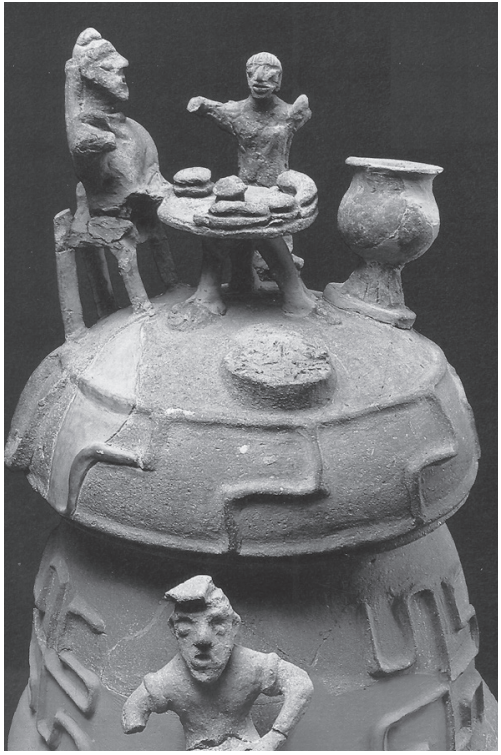

Fig. 3. Couvercle de l'ossuaire de Montescudaio. D'après G. Pugliese Carratelli (dir.), Rasenna, storia e civiltà degli Etruschi, Milan, 1986, p. 376, fig. 218. sans son épouse, devant une table tripode. À côté de lui se tient une servante avec un éventail. Près d'elle, un grand dinos sur un pied, probablement destiné à contenir le vin mélangé d'eau ${ }^{7}$. Il reste la base d'un autre élément brisé à la droite de l'homme. On y restitue le plus souvent un autre vase, mais la présence de deux grands dinoi qui auraient une fonction de cratère ne semble pas pertinente. Il faudrait plus certainement replacer une "corbeille», ou une table d'offrande de forme cylindrique semblable à celle de pierre qui se trouve près des trônes dans la tombe des Cinq sièges de Cerveteri ${ }^{8}$, celles de la tombe Campana I ou celles au fond de la tombe des Lions peints, encore à Cerveteri ${ }^{9}$. Il faut souligner que ces trois tombes sont contemporaines de notre vase. Sur la table tripode fixée sur le couvercle de l'ossuaire de Montescudaio, les mets paraissent être des galettes ou plutôt des pains de formes variées. Nous ne sommes pas

7 M. R. Lucidi, A. Mandolesi, " Gli Etruschi e il cibo », in C. CAsi, La cucina nel mondo antico. Archeologia e storia dell'alimentazione dalla preistoria al Medioevo, Pitigliano, 2009, p. 23-40 (p. 29-30).

8 S. SteingräвER, “ Überlegungen zu etruskischen Altären », in Miscellanea archaeologica Tobias Dohrn dedicata, Rome, 1982, p. 103-116, pl. 7, 3; S. SteIngräвer, “ Le culte des morts et les monuments de pierre des nécropoles étrusques ", in D. BriQuel, F. Gaultier, "Les plus religieux des hommes »(Paris, Rencontres du Louvre 1992), Paris, 1997, p. 97-116 (p. 101-102) ; F. Prayon, « Zum ursprünglichen Aussehen und zur Deutung des Kultraumes in der Tomba delle cinque sedie bei Cerveteri », Marburger Winckelmann-Programm, 1974, p. 1-15 ; F. Prayon, "Frühetruskische Grab- und Hausarchitektur ", Mitteilungen des Deutschen Archäologischen Instituts. Römische Abteilung. Ergänzungshefte, 22, Heidelberg, 1975, pl. 60-2, 61 ; G. ColonNA, “ Il dokanon, il culto dei Dioscuri e gli aspetti ellenizzanti della religione dei morti nell'Etruria tardoarcaica ", in Studi in memoria di S. Stucchi, Rome, 1996, p. 177-180. Principi etruschi, p. 169. M. Torelli (dir.), Gli etruschi, (catalogue de l'exposition du Palais Grassi de Venise 2000), Rome, 2000, p. 342 ; L. Нugot, Recherches sur le sacrifice en Étrurie (vII ${ }^{e}$ $I^{e r} s$. avant J.-C.), thèse de doctorat de l'université de Nantes, 2003, p. 65-68.

9 S. SteINGräвer, Überlegungen zu etruskischen Altären, p. 113, pl. 7, 2 ; S. Steingräber, Le culte des morts, p. 101-102 et 104 ; F. Prayon, Frühetruskische Grab, p. 112-113, pl. 62-63. 
dans le cadre strict du symposion, mais d'un banquet pendant lequel on buvait probablement du vin en mangeant en particulier des pains qui devaient être la base de l'alimentation des aristocrates comme des humbles. C'est le mode de vie aristocratique qu'on a voulu mettre en valeur ici. Il s'agit d'une interprétation locale du banquet oriental qui mélange les apports grecs (le vin mélangé d'eau) et une nourriture abondante. Nous sommes dans un contexte funéraire, mais la réalité quotidienne ne devait pas être très différente. On trouve parfois dans des tombes contemporaines des tables tripodes ${ }^{10}$ et des trônes qui montrent qu'on pouvait reconstituer dans des tombes ce type de banquet et déposer des offrandes sur les tables. La tombe Lippi 85, de Verruchio ${ }^{11}$ (650-625 av. J.-C.), dans une zone étrusquisée de la façade adriatique de l'Italie, contenait un matériel très riche. En plus d'un trône de bois, se trouvaient trois petites tables tripodes également en bois sur lesquelles avaient été déposés des plats. Sur l'une se trouvaient des noisettes, des pépins de raisin, des noyaux de cornouiller mâle ; sur une autre table, un service pour le vin et sur la troisième, des coupes hémisphériques dans lesquelles avaient été déposés du lièvre et du poisson dont on a retrouvé les os et des arêtes. Nous avons ici clairement trois catégories différentes d'offrandes : l'une avec des fruits, le nécessaire pour la consommation du vin sur une seconde table et les produits animaux sur une troisième. Par ailleurs, la sépulture contenait aussi quantité de vestiges carpologiques ${ }^{12}$. La complexité de l'organisation spatiale de cette tombe témoigne de la richesse des rituels funéraires dès l'époque orientalisante.

Le modèle qui s'impose en Étrurie, à partir de la fin du VII ${ }^{\mathrm{e}}$ s. av. J.-C., est ce qu'on nomme le banquet couché. La première attestation se trouve encore sur le couvercle d'une urne cinéraire, retrouvée dans une tombe à caisse de pierre à Tolle ${ }^{13}$ et conservée au Musée communal de Chianciano Terme où on trouve

10 Principi etruschi, p. 194, p 202.

1 P. von Eles (dir.), Guerriero e sacerdote. Autorità e comunità nell'età del ferro aVerucchio. La tomba del trono, Quaderni di Archeologia dell'Emilia Romagna 6, Florence, 2002 ; Principi etruschi, p. 365-372.

12 On a déposé comme offrandes quantité de restes végétaux : des grains de céréales, orge (Hordeum vulgare), blés (Triticum dicoccum, T. aestivum/durum) du millet commun (Panicum miliaceum), des graines de légumineuses, de la fève (Vicia faba), de la vesce commune (Vicia sativa) ainsi que de la lentille (Lens culinaris), diverses espèces de fruits, du raisin (Vitis vinifera), des pommes ou des poires (Malus/Pyrus), des noisettes (Corylus avellana), des cornouilles (Cornus mas) et des prunes (Prunus sp.), un légume: la gourde calebasse (Lagenaria siceraria) (Rottoli com. pers.).

13 G. Paolucci, “ Prime considerazioni sulla necropoli di Tolle presso Chianciano Terme », Annali della fondazione per il Museo "Claudio Faina” 7 (2000), p. 228-229 ; Lucidi, Mandolesi, Gli Etruschi e il cibo, p. 33, fig. 16. ThesCra (Thesaurus cultus et rituum antiquorum) II, 4.a., p. 263, n 2. 


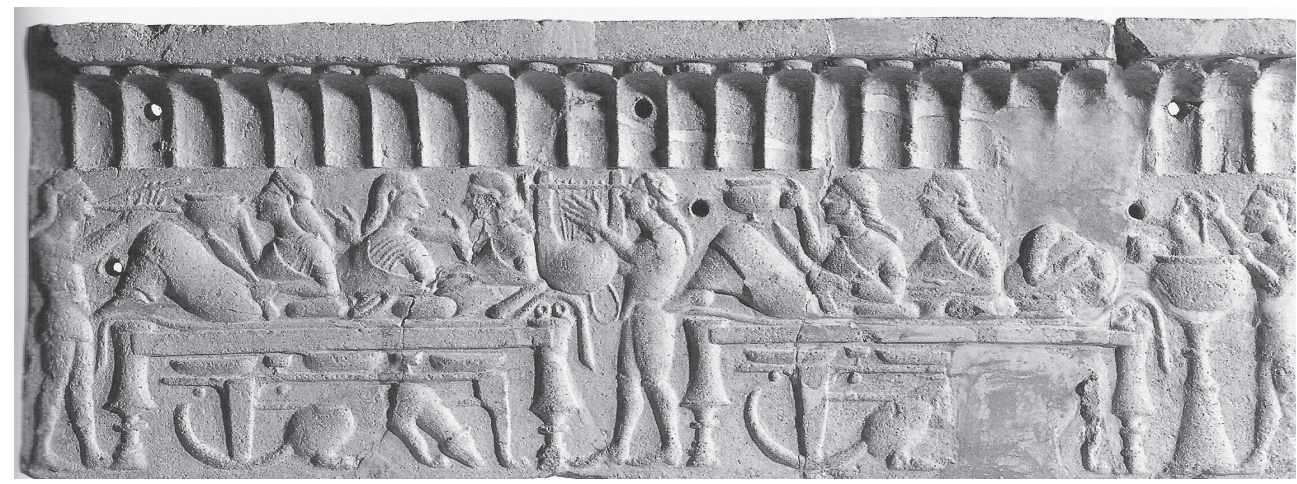

Fig. 4. Plaques de terre cuite architectoniques, Acquarossa. D’après G. Pugliese Carratelli (dir.), Rasenna, storia e civiltà degli Etruschi, Milan, 1986, p. 601, fig. 511.

simplement un homme couché. Il existe ensuite des milliers de représentations du banquet ou du symposion en Étrurie. Les convives peuvent être couchés sur des klinai ou sur des matelas posés à même le sol. La différence majeure avec les représentations grecques tient au fait que les femmes de l'élite sont souvent présentes sur les couches au côté des hommes.

Diodore de Sicile $(\mathrm{V}, 40)$ et Théopompe (frag. 222) ont relayé les poncifs qui faisaient à leur époque état de la propension des Étrusques à faire dresser deux fois par jour des tables opulentes et à se goinfrer avec leurs épouses. La documentation iconographique ne nous permet pas de confirmer ou d'infirmer leurs assertions. À plusieurs reprises, des artistes ont pris le soin, pour indiquer qu'il s'agissait d'un banquet et non d'un symposion, de placer sur les tables des vases aux formes ouvertes, destinés à contenir des mets. C'est ce qu'on observe sur des plaques de terre cuite architectoniques qui décoraient le palais de Murlo (580-570 av. J.-C.), à Acquarossa (560-550 av. J.-C.), Velletri ou encore Cerveteri (575-550 av. J.-C. $)^{14}$. On a des hommes et souvent aussi des femmes (même s'il est parfois difficile d'en avoir la certitude). Ces plaques évoquent un banquet civique, nous ne sommes pas en contexte funéraire. À Acquarossa, les convives sont trois par couche. Ceux situés à l'extrémité gauche des klinai tiennent une coupe kylix et une machaira qui est une allusion claire au sacrifice. Les trois grands

14 J.P. Small, “ Eat, drink, and be merry. Etruscan banquets », in Murlo and the Etruscans. Art and society in ancient Etruria, Madison Wisc., 1994, p. 85-94. L'auteur voit dans les représentations de Murlo un banquet public et non un simple symposion. A Murlo et Velletri elle identifie à coup sûr des femmes (p. 87). A. Rathse, “ Banquet and ideology. Some new considerations about banqueting at Poggio Civitate ", in Murlo and the Etruscans, 1994, p. 95-99. 


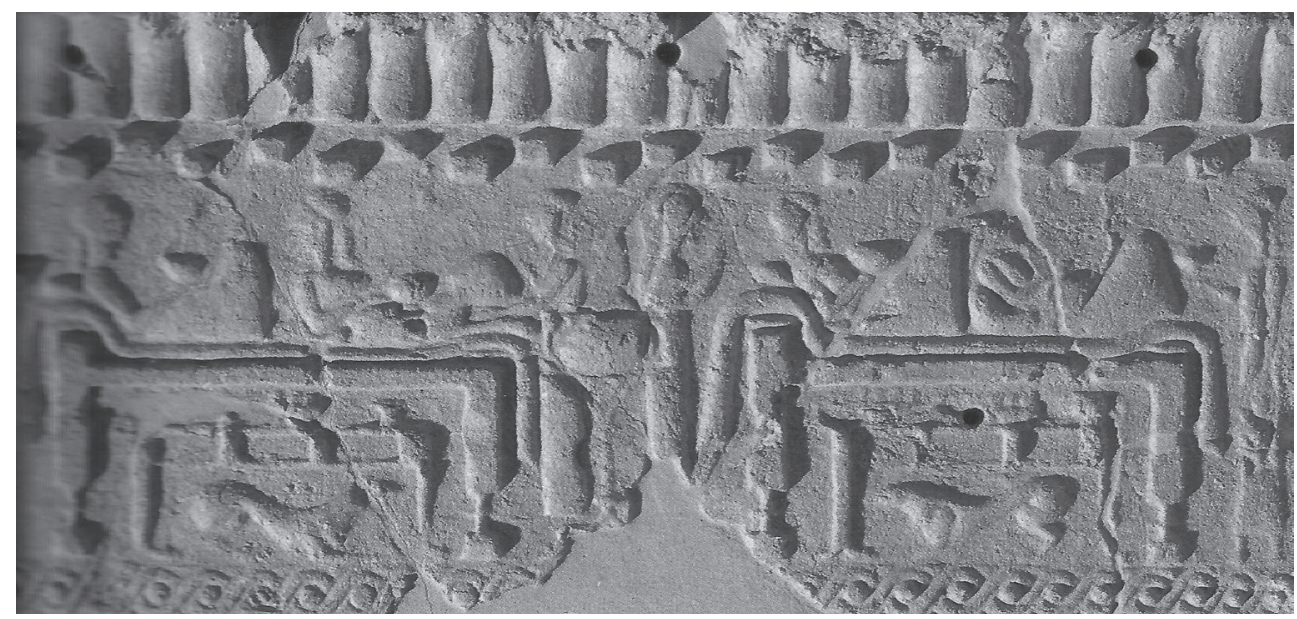

Fig. 5. Plaques de terre cuite architectoniques, Murlo. D'après G. Pugliese Carratelli (dir.), Rasenna, storia e civiltà degli Etruschi, Milan, 1986, p. 405, fig. 298.

plats et les deux petites coupes vides qui sont posées sur les tables montrent que nous sommes dans le cadre du banquet et non du symposion (fig. 4). Cette simple allusion était suffisante, les artistes n'ont pas jugé utile d'être plus précis sur la nature des mets. La présence de femmes au banquet est plus assurée sur les plaques de Murlo où cette fois les aliments sont sommairement représentés (fig. 5). Il est impossible de définir la nature des mets qui se trouvent dans les deux plats posés sur les tables : petits œufs, fruits, biscuits? En tout cas, l'un des convives, celui qui se retourne sur la couche de droite, tient une coupe dans sa main droite et un petit bâtonnet allongé qu'il vient de prendre dans un plat dans son autre main. P. Marinval a émis à ce sujet l'hypothèse de petits biscuits qu'on pouvait tremper dans le vin pour les ramollir ${ }^{15}$. Ce n'est pas le couple qui est mis en valeur, mais la pratique sociale du banquet collectif dans un contexte civique. On retrouve aussi des allusions à ces banquets collectifs en contexte funéraire, comme dans la tombe du Triclinium de Tarquinia (470-460 av. J.-C.) où des plats vides sur les tables évoquent la nourriture. Toutefois, ce sont les représentations du symposion de tradition attique qui s'imposent au $\mathrm{V}^{\mathrm{e}} \mathrm{s}$. av. J.-C. Il ne s'agit plus de manger, mais seulement de boire et la presque totalité des représentations montre des tables destinées principalement à recevoir les coupes des convives lorsque ceux-ci voulaient soulager un peu leur bras, comme on le voit sur les

15 D. Frère, L. Нugot, P. Marinval, Le végétal offert et consommé dans les rituels funéraires étrusques, Communication au congrès du CTHS, Rennes, 2014. http://wikiradio.ueb. eu/channel/1_UEB\#podcast (site consulté le 17 février 2016). 
représentations grecques, en particulier dans la tombe du Plongeur de Paestum ${ }^{16}$. Les tables, lorsqu'elles sont figurées, sont le plus souvent vides. On trouve donc peu de représentations de couples au banquet pendant toute la période archaïque et classique, ce sont les représentations du symposion qui dominent largement.

Si on recherche des programmes figuratifs clairement centrés sur le couple fondateur de la tombe et pas sur les agapes collectives comme dans la tombe des Boucliers, il faut se pencher sur les peintures funéraires de Tarquinia de la fin du $\mathrm{VI}^{\mathrm{e}}$ s. av. J.-C., avant que ne s'impose le style attique. Sur la paroi du fond de la tombe du Vieux ${ }^{17}$, dans la tombe des Vases peints ${ }^{18}$ et sur le fronton de la chambre du fond de la tombe de la Chasse et de la Pêche ${ }^{19}$, c'est l'union de deux êtres qui est évoquée. L'attitude et les gestes des personnages couchés renvoient explicitement à l'exaltation du couple conjugal dans sa connotation gentilice ${ }^{20}$. La famille est née de cette alliance. La femme offre une couronne à son époux tandis que celui-ci présente une grande coupe devant elle dans laquelle se trouve le liquide, probablement le vin, qu'ils vont partager. Dans la tombe de la Chasse et de la Pêche, le banquet se déroule dans une atmosphère parfumée, puisque le petit esclave qui se trouve derrière l'homme est en train de puiser du parfum dans un alabastre qui n'est malheureusement plus visible, à l'aide d'une fine tige dont on voit encore l'extrémité dans sa main. On doit probablement restituer un alabastre dans la main des femmes sur le sarcophage des époux de la Villa Giulia, semblable à celui de la petite urne Cp 5193 conservée au Louvre ${ }^{21}$. C'est moins clair sur le sarcophage du Louvre, on pourrait y restituer une grenade, mais il pouvait aussi s'agir d'un vase à parfum, un aryballe. L'homme sur le sarcophage des époux du Louvre a perdu sa main, mais elle devait être ouverte comme sur le sarcophage de la Villa Giulia. Il est possible qu'une coupe ait été à l'origine posée dans cette main ${ }^{22}$ ou que la femme ait versé de l'huile parfumée dans le creux de

16 M. NAPoli, La Tomba del Tuffatore. La Scoperta della grande pittura greca, Bari, 1970 ; A. Pontrandolfo, A. Rouveret, Le Tombe dipinte di Paestum, Modène, 1992 ; R. Ross Holloway, " The Tomb of the Diver ", American Journal of Archeology 110-3 (juillet 2006), p. 365-388.

17 S. Steingräвer, Catalogo ragionato, p. 363.

18 S. Steingräвer, Catalogo ragionato, p. 361-362.

19 S. Steingräвer, Catalogo ragionato, p. 301-302.

20 R. Benassai, “ Per una lettura del programma figurativo della Tomba delle Bighe di Tarquinia », Orizzonti 2 (2001), p. 51-62.

21 Voir aussi la main de terre cuite qui tient un alabastre du musée de Bruxelles: F. Gaultier, L. Haumesser (dir.), Les Étrusques et la Méditerranée, la cité de Cerveteri, (catalogue de l'exposition du Louvre-Lens 2013), Paris, 2013, p. 187, n 192.

22 M.-F. Briguet, Le sarcophage des époux de Cerveteri du Musée du Louvre, Paris, 1988 ; M.-F. Briguet, Le sarcophage des époux de Cerveteri du Musée du Louvre, Florence, 1989 (Monumenti Etruschi 4). 
la main de son époux ${ }^{23}$. Nous sommes dans un contexte évoquant le lien matrimonial tissé entre deux êtres, entre deux familles, pour créer une nouvelle entité.

Sur un miroir conservé au Musée de la Villa Giulia et daté du Ve s. av. J.-C., on retrouve les mêmes éléments ${ }^{24}$. La femme tient une couronne et un objet, sans doute un oiseau, est posé dans son autre main. Il pourrait s'agir d'un présent érotique, comme celui que pouvait offrir en Grèce un éraste à son éromène (fig. 6). Cela ne serait pas déplacé sur un miroir. Ainsi, dans la tombe des Vases peints, un jeune garçon nu est assis sur les genoux d'une jeune femme, son bras passé dans le dos de celle-ci. Il s'agit d'une scène de séduction. Tandis qu'elle lui tend une couronne, l'homme lui présente un petit oiseau qu'il tient dans sa main droite. Mais revenons au

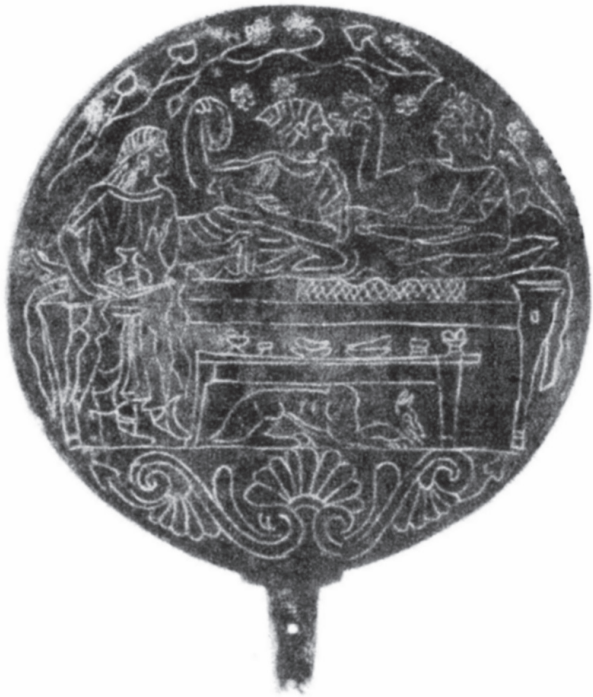

Fig. 6. Miroir de bronze conservé à Rome, Musée de la Villa Giulia. D’après L. C. Pieraccini, « Families, Feasting and Funerals: Tomb Practice at Ancient Caere », Etruscan Studies 7 (2000), p. 36, fig. 1. miroir de bronze du Musée de la Villa Giulia. Le caractère érotique de la scène est conforté par le fait que l'homme couronné qui présente une grande coupe dans sa main gauche tient une fleur qu'il approche du visage de la femme dans son autre main. Il existe plusieurs représentations de ce type comme dans la tombe 5513 de Tarquinia $^{25}$. Nous sommes bien ici dans le cadre d'un banquet, car des victuailles sont représentées sur la table qui se trouve devant la couche. Malheureusement, les mets sont figurés trop sommairement pour qu'on puisse savoir s'il d'agissait de biscuits, de viandes, de pains, de galettes, de fruits ou d'œufs.

23 Nous renvoyons sur ce sujet aux études de Dominique Frère. Voir par exemple D. Frère, “ Gestes quotidiens et parfum d'immortalité », in L. Bodiou, D. Frère, V. MeHL, L'expression des corps. Gestes, attitudes, regards dans l'iconographie antique, Rennes, 2006, p. 195-212 et idem, “ Démons parfumés en Étrurie, de l'époque orientalisante », in J.-P. Boudet, P. Faure et C. Renoux (dir.), De Socrate à Tintin. Anges gardiens et démons familiers de l'Antiquité à nos jours, Rennes, 2011, p. 21-30 (p. 26-27).

24 L. C. Pieraccini, "Families, Feasting and Funerals: Tomb Practice at Ancient Caere », Etruscan Studies 7 (2000), p. 35-49 (p. 36, fig. 1).

25 S. Steingräber, Catalogo ragionato, p. 377. 
La mise en valeur du couple fondateur était essentielle pour la structuration des gentes étrusques, certainement déjà depuis l'époque orientalisante. Ce désir d'exalter le couple créateur de la famille ne s'éteint pas à la période classique, même si l'iconographie le montre peu. Les deux cônes ornés placés sur une énorme kliné dans la tombe du Lit funèbre pourraient être la représentation symbolique des fondateurs de la famille, selon l'hypothèse de J.-R. Jannot ${ }^{26}$. Le symposion qui se déroule autour aurait pour fonction la célébration de ce couple, témoin et fondateur de la solidarité gentilice. Pour C. Isler-Kerényi le doublement des vases dans certaines tombes de l'époque classique, en particulier à Spina, serait à mettre en relation avec cette volonté d'indiquer qu'on n'honore pas une personne, mais bien un couple ${ }^{27}$.

Après une longue période pendant laquelle cela ne semble plus essentiel, on perçoit dans plusieurs tombes la volonté de recentrer l'attention sur le couple à partir de la seconde moitié du $\Gamma^{\mathrm{e}} \mathrm{s}$. av. J.-C., de réaffirmer son importance, probablement du fait de la crainte de la dissolution des familles dans cette époque troublée. Ainsi, dans la tombe du Triclinium de Cerveteri ${ }^{28}$, datée de la fin du $\mathrm{IV}^{\mathrm{e}} \mathrm{s}$. av. J.-C. on constate qu'on n'est pas réellement en présence d'un banquet collectif, mais de la répétition à neuf reprises d'une même scène. Un couple composé d'un homme et d'une femme est allongé devant une table ronde à trois pieds chargée de viandes, de fruits, d'œufs, de gâteaux et de vases selon la description de J. Martha (dans L'art étrusque, Paris, 1889). Cette fois, il s'agit clairement d'un ensemble de couples qui mangent les uns auprès des autres et non d'un banquet collectif. L'affirmation du couple dans les banquets par rapport aux agapes collectives précédentes est patente. Sur la paroi du fond de la tombe, deux couples situés de part et d'autre d'un extraordinaire service à boire semblent plus importants que les autres. Les peintures de cette tombe sont malheureusement très dégradées. Il ne nous reste qu'une description de Jules Martha de la fin du XIX ${ }^{\mathrm{e}}$ s. et un dessin approximatif de Luigi Canina ${ }^{29}$. On ne peut ainsi pas clairement identifier les mets présents sur les tables.

Les peintures de la tombe des Boucliers de Tarquinia qui datent de la même époque sont heureusement mieux conservées (fig. 1 et 2). Le programme iconographique est encore organisé autour de plusieurs couples. La gestuelle des parents de Larth, leur attitude, met en évidence leur intimité. Il ne s'agit pas d'un

26 J.-R. JANnot, “Une représentation symbolique des défunts », Mélanges de l'École Française de Rome. Antiquité 89-2 (1977), p. 579-588.

27 C. Isler-KerÉnYI, “ Images grecques au banquet funéraire étrusque », Pallas 61 (2003), p. 39-53, émet cette hypothèse à propos de la tombe Valle Trebba 128 de Spina.

28 Steingräвer, Catalogo ragionato, p. 273.

29 L. CANina, L'antica Etruria Marittima, I, Rome, 1846, p. 194, pl. 63-64. 
banquet traditionnel, car si l'homme est à demi allongé, la femme est, elle, assise à ses pieds, dans une position qu'on retrouve fréquemment sur les couvercles d'urnes cinéraires à la même époque. On pourrait y voir une évolution dans les rapports entre hommes et femmes, car si l'homme pose délicatement sa main droite sur l'épaule de son épouse et lui présente une belle coupe qui symbolise leur union, celle-ci ne partage pas sa couche et elle a une attitude réservée. Si l'intimité des deux est perceptible, on est bien loin des couples de la fin du $\mathrm{VI}^{\mathrm{e}} \mathrm{s}$. av. J.-C. qui croisaient leurs regards et partageaient la même couche. Larth a avec son épouse la même attitude que ses parents, mais c'est elle qui cette fois pose la main sur l'épaule de son mari. Il faut noter la présence d'une femme avec un grand éventail auprès du couple, comme sur l'ossuaire de Montescudaio, même s'il ne faut se garder d'en tirer des conclusions hâtives en raison du hiatus chronologique. Ce n'est pas une coupe qui symbolise leur union, mais l'échange d'un petit objet sphérique blanc qui doit être un œuf. C'est un élément important et plusieurs chercheurs s'y sont intéressé : C. Guarnieri pour Spina, J. P. Small et L. C. Pieraccini dans plusieurs articles ${ }^{30}$. On trouve fréquemment des œufs en contexte funéraire. Sur de nombreux reliefs archaïques de Chiusi, des hommes ou des femmes tiennent ce que J. R. Jannot identifie à un œuf. Par exemple, sur un relief de Berlin, parmi les trois banqueteurs, la femme au centre en tiendrait $u^{31}$. On en trouve aussi la représentation dans plusieurs tombes. Dans la tombe

30 C. Guarnieri, “ La presenza dell'uovo nelle sepolture di Spina (Valle Trebba). Un problema aperto ", in Studi sulla necropoli di Spina in Valle Trebba, (colloque de Ferrare du 15 octobre 1992), Ferrare, 1993, p. 181-195 ; P. Small, Eat, Drink, and be Merry, p. 85-89 ; L. C. Pieraccini, Around the Hearth : Caeretan Cylinder-Stamped Braziers, Rome, 2003; Pieraccini, Families, p. 35-49 ; ead., "L'inafferrabile uovo etrusco », in M. D. Gentili (éd.), Studi e ricerche a Tarquinia e in Etruria (séminaire international en souvenir de Francesca R. Serra Ridgway), Tarquinia, 2013, p. 105-125 ; idem, “ Food and Drink in the Etruscan World ", in J. Turfa, The World of the Etruscans, New York, 2013, p. 812-822.

31 J.-R. Jannot, Les reliefs archaïques de Chiusi, Collection de l'École française de Rome 71, Rome, 1984, C.II.42, de la Collezione Mazetti. Voir aussi sur ICAR: http://icar.huma-num.fr/icardb/scene.php?idscene=BERL442b (site consulté le 17 février 2016). Même chose sur la face droite? http://icar.huma-num.fr/icardb/scene. php?idscene=BERL442c (site consulté le 17 février 2016). Peut-être aussi Jannot, Les reliefs archaïques de Chiusi, D. I. 5, Collezione Mazetti (http:/icar.huma-num.fr/icardb/ scene.php?idscene=BERL463c\&idsupport=BERL463 (site consulté le 17 février 2016)) ; idem, Les reliefs archaïques de Chiusi, D' inclassable 2, sarcophage Blayds (http://icar. huma-num.fr/icardb/scene.php?idscene=BLAY498a (site consulté le 17 février 2016)); idem, Les reliefs archaïques de Chiusi, C.III.6, Chiusi, Museo civico ; idem, Les reliefs archaïques de Chiusi, D. II. 11, Copenhague, Glyptothèque Ny-Carlsberg; idem, Les reliefs archaïques de Chiusi, C.III.17, Firenze, Museo archeologico ; idem, Les reliefs 
des Lionnes ${ }^{32}$, un homme tient un petit œuf dans sa main droite. Un homme présente un objet sphérique qui pourrait être un œuf à une femme dans la tombe des Léopards ${ }^{33}$. Il pourrait également y en avoir sur les tables tripodes de la tombe Golini I selon S. Steingräber ${ }^{34}$. Sur les fresques de la tombe de la Pucelle, la femme sur le lit de droite pourrait offrit petit un ouf à son conjoint ${ }^{35}$. On pourrait multiplier les exemples ${ }^{36}$. La difficulté tient au fait qu'il existe un grand nombre de formes et de tailles d'œufs dans la nature et qu'il est souvent compliqué de les reconnaître avec certitude.

En Étrurie, on a retrouvé plusieurs œufs conservés dans des tombes. Récemment encore dans une olla dans une petite sépulture à chambre hellénistique, la Tomba delle tre uova à Castello di Casenovole (Civitella Paganico). Il s'agirait d'œufs de poule, préservés par des dépôts calcaires selon M. A. Turchetti à qui on doit cette découverte. Des études plus précises sont en cours pour savoir s'ils ont été déposés cuits ou crus ${ }^{37}$. Les œufs sont fragiles et leur conservation est exceptionnelle. Par chance, plusieurs ont été conservés dans un brasero cérétain retrouvé dans la tombe Maroi III. Ce brasero n'a pas été vidé après sa découverte et il est aujourd'hui conservé, avec son contenu au Musée de la Villa

archaïques de Chiusi, D.II.10, Collezione Castellani; idem, Les reliefs archaïques de Chiusi, C.I.33, Palermo, Museo Nazionale ; idem, Les reliefs archaïques de Chiusi, C.I.36, Palermo, Museo Nazionale ; Jannot C.I.41, Paris, Musée du Louvre ; idem, Les reliefs archaïques de Chiusi, D.I.10, Collection Campana ; idem, Les reliefs archaïques de Chiusi, B'.II.2, Roma, American Academy ; idem, Les reliefs archaïques de Chiusi, C.I.5, Siena, Museo archeologico.

32 SteingräBer, Catalogo ragionato, p. 324-325.

33 SteingräBer, Catalogo ragionato, p. 327.

34 Steingräвer, Catalogo ragionato, p. 286.

35 Voir http://icar.huma-num.fr/icardb/scene.php?idscene=TARQ15b (site consulté le 17 février 2016); E. Brizıo, “Tombe dipinte di Corneto », Bullettino dell'Instituto di corrispondenza archeologica 45 (1873), p. 73-85, 97-107, 193-204.

36 Tomba dei Demoni azzurri (tombe des Démons bleus), G. Adinolfi, R. Carmagnola, M. Cataldi, "La Tomba dei demoni azzurri. Le pitture », in Pittura parietale, pittura vascolare. Ricerche in corso tra Etruria e Campania, (actes de la journée d'études de Santa Maria Capua Vetere 28 mai 2003), Naples, 2005, p. 45-72. Tomba delle Bighe (tombe des Biges), Steingräвer, Catalogo ragionato, p. 297-299. Tomba del Frontoncino (tombe du petit Fronton), Steingräber, Catalogo ragionato, p. 314. Tomba 5513, Steingräber, Catalogo ragionato, p. 377.

37 M. A. Turchetti, F. Cavalli, S. Cencetti et alii, « La Tomba delle tre uova di Casenovole (Civitella Paganico, GR). Qualche considerazione su offerte funebri, riti di passaggio e nuove metodologie non distruttive di indagine ", in Amore per l'antico. Dal Tirreno all'Adriatico, dall preistoria al medioevo e oltre. Studi di antichità in ricordo di Giuliano de Marinis, Rome, 2014, p. 169-182. 
Giulia $^{38}$. Dans le cadre du programme MAGI financé par l'Agence Nationale de la Recherche (Manger, boire, offrir pour l'éternité en Gaule et Italie préromaines. Archéologie des offrandes funéraires de produits biologiques dans les cultures celtes, étrusques, italiques et phénico-puniques [ $\mathrm{VI}^{\mathrm{e}} \mathrm{s}$. $-\mathrm{I}^{\mathrm{er}} \mathrm{s}$. av. J.-C.]), nous avons constitué une équipe pluridisciplinaire (archéologue, palinologue, paléogénéticien, carpologue, archéozoologue) pour étudier les restes contenus dans les braseros conservés à Rome et à Cerveteri ${ }^{39}$. Il est possible que de minuscules traces de coquilles soient conservées dans d'autres braseros, parmi les cendres et des études morphologiques et paléogénétiques devraient bientôt nous permettre d'en savoir beaucoup plus sur ces œufs. Il reste à comprendre si on doit voir une symbolique funéraire dans la présence d'un œuf dans la main de la femme de Larth Velcha ou s'il était l'évocation de la fertilité du couple.

Outre cet œuf, beaucoup d'autres mets sont représentés dans la tombe des Boucliers. Les deux couples de défunts ne semblent pourtant pas être des banqueteurs. Il faudrait ainsi plutôt voir des offrandes sur les tables. La découverte dans plusieurs tombes de petites tables tripodes déjà évoquées ainsi que les deux tables de pierre situées devant les sièges sur lesquels étaient disposés les ancêtres familiaux dans la tombe des Cinq sièges de Cerveteri montrent que cette pratique était ancienne. Les représentations de la tombe Golini I évoquent par contre plus certainement la préparation d'un véritable banquet funéraire. On retrouve des galettes et peut-être des œufs, mais aussi beaucoup d'autres aliments comme du raisin, des préparations à base de viande en quantité, des grenades, la préparation d'un brouet...

Les offrandes présentées sur les tables dans la tombe des Boucliers n’ont pas été choisies au hasard, elles avaient une importance symbolique. On ne peut pas définir ce que représente la masse informe située au centre, mais sur les côtés, il s'agit de gros pains. Soit deux pains posés l'un sur l'autre, soit selon l'hypothèse de P. Marinval un pain plié comme ceux qui ont été retrouvés à Herculanum. Les petites boules situées à côté sont tour à tour décrites comme des gâteaux, des olives, des raisins blancs ou encore de petits œufs. En tout cas, ils sont posés à même la table. P. Marinval a émis l'hypothèse séduisante d'un pain de type rosetta à propos de l'un d'entre eux, mais pour les trois autres, il est difficile de se faire une idée précise ${ }^{40}$.

38 Pieraccini, Around the Hearth, p. 46 (A4.09) ; M. Moretti, Il Museo Nazionale di Villa Giulia, Rome, 1962, 93f, fig. 72. Il est plein de charbons. Il contient aussi un tisonnier de bronze, deux canthares de bucchero, un kylix et des œufs. Photo dans Pieraccini, Around the Hearth, p. 46, fig. 10.

39 Nous remercions Madame Alfonsina Russo et Madame Rita Cosentino qui ont l'amabilité de nous permettre de collaborer avec elles pour faire cette étude.

40 Frère, Hugot, Marinval, Le végétal offert et consommé dans les rituels funéraires étrusques. 
Si ces mets étaient déposés en l'honneur des défunts dans les nombreux plats trouvés dans les tombes, ils n’ont le plus souvent laissé aucune trace, car ces éléments organiques ont été rapidement détruits. Toutefois la présence d'œufs dans au moins l'un des braseros de Cerveteri est pour nous porteuse d'espoir. Le pain, comme les œufs ont pu être déposés et brûlés ou cuits dans ces braseros, ce qui aurait assuré leur conservation. Une étude fine à la loupe binoculaire des cendres contenues dans les braseros nous permettra de savoir si des restes de pain ou de galettes se trouvent parmi les cendres. Le savoir serait une avancée importante dans les études sur l'alimentation en Étrurie. À ce jour, seuls quelques fragments calcinés de " pain » ont été retrouvés dans la tombe Lippi 85 de Verruchio $^{41}$. Comme ils n'ont pas encore fait l'objet d'étude, il n'est pas possible de préciser leur nature exacte : pain ou galette ainsi que la composition de la pâte.

Les offrandes faites dans les tombes ne se limitaient pas au pain et aux œufs. Dans les anciens rapports de fouilles, on retrouve de nombreuses mentions d'aliments décomposés. Depuis, les techniques ont évolué et on dispose de plus en plus d'informations intéressantes. Dans la tombe A de la nécropole orientalisante de Casa Nocera ${ }^{42}$ à Casale Marittimo, dans le Val di Cecina, datée du premier quart du VII s. av. J.-C. on a retrouvé trace de noisettes, de pommes, de raisins, d'un rayon de ruche ainsi que des pépins de grenades qui comme les œufs apparaissent dans l'alimentation à cette époque. Dans la même nécropole, la tombe G contenait raisins, noix et grenades. Dans la tombe des Olives de Cerveteri (575-550 av. J.-C.), de nombreux noyaux d'olives (Olea europaea) étaient déposés dans une sorte de braseros ${ }^{43}$. Des pommes de pin pignon (Pinus pinea) ont aussi été déposées dans un bol dans une tombe explorée à Vulci, dans la nécropole de l'Osteria, au début des années 2000. On pourrait encore donner des exemples d'ossements et d'arêtes de poissons qui ont aussi parfois été conservés dans des contextes funéraires. Si la symbolique funéraire des grenades et des pommes de pin est claire, il reste encore beaucoup de travail à faire pour comprendre le choix des autres dépositions : partage des viandes de sacrifices, offrandes ou partage du banquet funèbre.

41 Sur cette tombe, voir Principi etruschi, p. 365-372, n`524-566 ; G. Bermond Montanari, La formazione della città in Emilia Romagna, Bologne, 1987 ; M. Forte (dir.), Il dono delle Eliadi. Ambre e oreficie dei principi etruschi di Verucchio, (catalogue de l'exposition du Musée de Verucchio 1994), Rimini, 1994.

42 A. M. Esposito (éd.), Principi Guerrieri. La Necropoli Etrusca di Casale Marittimo, Milan, 2001, p. 39-56.

43 P. Giulierini, “ I piaceri della tavola: il mondo femminile e la cucina », in G. Paolucci (dir.), Larthia. La vita di una donna al tempo degli Etruschi, (acte du colloque de Chianciano Terme 2007), Florence, 2008, p. 27-63 (p. 32) ; L. Aмвrosini, “ Una lekanis etrusca a figure rosse ", Mélanges de l'École française de Rome. Antiquité, 125-1 (2013), fig. 22, http://mefra.revues.org/1273 (site consulté le 17 février 2016). 
Après une longue éclipse, entre la fin du VIe s. av. J.-C. et le IVe s. av. J.-C., les représentations des couples fondateurs de la lignée familiale réapparaissent en contexte funéraire, mais dans un contexte idéologique et social différent comme en témoignent les positions des époux sur les couches. Les deux représentations de couples de la tombe des Boucliers en sont un témoignage. Les mets qui ont été déposés sur les tables de bois dressées devant les convives ne sont pas les aliments d'un banquet comme celui représenté dans la tombe Golini I, mais des offrandes faites aux défunts. Parmi ces offrandes, il est intéressant de constater que les pains, qu'on observe dans un contexte similaire à l'époque orientalisante, tiennent encore une place essentielle. On pourrait y voir une forme de permanence dans les offrandes faites aux défunts, même s'il faut rester prudent compte tenu des données chronologiques. Dans la tombe du Lit funèbre, selon F. Poulsen ${ }^{44}$, à droite de la grande kliné se trouvent deux symposiastes et deux esclaves tenant l'un un oeuf et l'autre un pain dans un geste d'offrande, deux éléments qu'on retrouve dans la scène de Larth et de son épouse dans la tombe des Boucliers. Les offrandes alimentaires étaient sans aucun doute essentielles dans le cadre des rituels funéraires, mais du fait de leur fragilité elles ont le plus souvent disparu. Les techniques nouvelles et une approche pluridisciplinaire pourront heureusement nous permettre des avancées spectaculaires dans la connaissance de ces rituels dans un futur proche.

\author{
Laurent Hugot \\ Maître de conférences \\ en histoire ancienne \\ Université de La Rochelle (flash) \\ 1, parvis Fernand Braudel \\ 17000 La Rochelle \\ laurent.hugot@univ-lr.fr
}

44 F. Poulsen, Etruscan tomb-paintings. Their subjects and significance, Oxford, 1922. 
